\title{
Retrospective analysis of the clinical behavior of oral hairy leukoplakia in 215 HIV-seropositive patients
}

\section{Daniela Assis do VALE(a) Fabiana Martins e MARTINS(a) Paulo Henrique Braz da SILVA ${ }^{(b)}$ Karem López ORTEGA(a)}

(a) Universidade de São Paulo - USP, Special Care Dentistry Center, Department of Oral Pathology, São Paulo, SP, Brazil.

(b) Universidade de São Paulo, Department of General Pathology, São Paulo, SP, Brazil.

Declaration of Interests: The authors certify that they have no commercial or associative interest that represents a conflict of interest in connection with the manuscript.

\section{Corresponding Author:}

Karem López Ortega

E-mail: klortega@usp.br

DOI: 10.1590/1807-3107BOR-2016.vol30.0118

Submitted: Mar 16, 2016

Accepted for publication: Aug 25, 2016

Last revision: Aug 31,2016
Abstract: Oral manifestations are common findings in human immunodeficiency virus (HIV) infected patients and frequently influence the overall health. Oral hairy leukoplakia (OHL) is strongly associated with HIV infection demonstrating its relationship with the individual's immune status and progression of immunosuppression. This study aims to retrospectively evaluate OHL in HIV patients, analyzing its incidence, demographic aspects and possible changes in clinical and epidemiological profile of the disease over 17 years. The records of 1600 HIV-infected patients were reviewed. The data were correlated and analyzed, considering HIV exposure category, age, gender, harmful habits, CD4 level, use and type of antiretroviral. OHL was observed in $215(13.4 \%)$ patients. Most were men in the fourth decade of life, $171(79.5 \%)$ and 112 (52,1\%) respectively, but an increase in the incidence of OHL among female patients and those in the fifth decade of life was observed. Tobacco smoking was the most frequent harmful habit reported by $114(68 \%)$ patients. OHL occurred mostly in patients with CD4 counts between 200 and 500 cells $/ \mathrm{mm}^{3} 35$ (55.5\%). The lower incidence of OHL was found among patients using at least one non-nucleoside reverse transcriptase inhibitor (NNRTI). OHL is related to CD4 count, use of ARVT and tobacco smoking and is also more prevalent in men in the fourth decade of life. These characteristics were recognized in absolute values, but when verifying the behavior over the years we noticed that the incidence of $\mathrm{OHL}$ is decreasing and its epidemiological characteristics changing.

Keywords: HIV; Acquired Immunodeficiency Syndrome; Leukoplakia, Hairy; Epidemiology.

\section{Introduction}

Human immunodeficiency virus (HIV) infection is considered as one of the major health threats of the world since 1981, when the infection was officially recognized by the Centers for Disease Control and Prevention (CDC). Since then, studies were conducted for better understanding of the nature of the infection, as incidence and development of opportunistic infections; and its relationship with clinical and demographic aspects ${ }^{1,2,3,4}$. One of the most common manifestations described in HIV-positive patients are the oral alterations, usually affecting the general health of these patients ${ }^{1}$, predicting a worse prognosis and representing an important 
clinical marker for disease progression ${ }^{2}$.There are many oral lesions that have been associated to HIV infection; those are classified into three groups according to their intensity and features ${ }^{3}$.

The first group is characterized by the oral lesions strongly associated with HIV, such as candidiasis, oral hairy leukoplakia (OHL), linear gingival erythema, acute necrotizing ulcerative gingivitis, necrotizing ulcerative periodontitis, Kaposi's sarcoma and non-Hodgkin lymphoma. The second group includes atypical ulcers, salivary glands disorders and viral infections including cytomegalovirus, herpes simplex, papillomavirus and varicella-zoster. Lastly, the third group consists of oral complications observed in HIV infection but not considered a distinctive lesion associated with the virus such as osteomyelitis, neurological disorders, sinusitis and squamous cell carcinoma, among others ${ }^{3}$.

OHL is almost exclusively seen in immunosuppressed patients ${ }^{5,6}$. The detection of Epstein-Barr virus (EBV) DNA in the epithelial cells is confirmatory for the diagnosis of this lesion ${ }^{5}$, OHL is clinically described as asymptomatic non-removable white patch with corrugated surface. This white lesion can be distinguished by the formation of vertical grooves most commonly seen on the sides of the tongue, often bilaterally $^{7}$. Clinical differential diagnosis is based on the exclusion of irritative hyperkeratosis, pseudomembranous candidiasis, lichen planus and white sponge nevus. The histopathological features of OHL are not pathognomonic, and include hyperkeratosis, epithelial hyperplasia, ballooning degeneration, acanthosis, and mild or moderate inflammatory infiltrate ${ }^{8}$.

The diagnosis can be established on a clinical basis, though some authors believe that the presence of EBV should be confirmed by in situ hybridization ${ }^{8,9}$. The importance of an accurate diagnosis of OHL relies in the fact that this condition has a clear prognostic value for HIV infection progression and is classified as a Centers for Disease Control and Prevention category-B clinical marker of HIV disease ${ }^{10}$. In HIV-seropositive patients OHL suggests a lower CD4 account and a higher viral load level ${ }^{2,11}$. OHL can be also an indicator of therapeutic failure or patient non-adherence to antiretroviral treatment ${ }^{12}$.
Some patients request treatment, which can be local, using keratolytic substances, or systemic with antiviral (eg. Acyclovir) or antiretroviral drugs, though it frequently resolves with highly active antiretroviral therapy (HAART), if associated with HIV infection ${ }^{13}$. However, since OHL does not represent a biological risk or have any symptoms, the treatment is unnecessary ${ }^{13}$.

OHL occurs mainly in adults, is uncommon in children, and affects mainly homosexual males. Most of the epidemiological studies places this lesion as the second most prevalent, but its incidence has been variable in different countries ${ }^{14,15}$.

The introduction of new antiretroviral therapies, such as those used in HAART protocols, caused marked reduction in the frequency of oral manifestations in general, but especially in those strongly associated with HIV, as candidiasis and OHL. Some authors also believe that changes in the clinical course and characteristics of those lesions can already been seen ${ }^{2,16}$.

The HIV epidemic also presented important changes in some countries, notably in the increment of the heterosexual HIV population ${ }^{17}$. It is possible that these changes could also affect the clinical and epidemiological behavior of oral manifestations, and the temporal assessment of large populations may be useful in identifying new patterns of these manifestations.

In the present retrospective study we analyzed the presence of OHL in HIV positive patients treated at Special Care Dentistry Center (School of Dentistry, University of São Paulo) over 17 years. Then, the incidence, demographics and possible changes in clinical and epidemiological profile of the disease were evaluated.

\section{Methodology}

The study protocol was approved by the Ethics Committee (protocol number: 78/99). The records of $1600 \mathrm{HIV}$-infected patients admitted at our institution from 1989 to 2006 were revised. At baseline visit clinical history was taken and oral examination was performed. The data collected at the patient's initial visit were transferred to a computer program developed by the CDC, Epi Info 6 version 6.04c, where the data was correlated and analyzed, considering the variables of HIV exposure category, age, gender, 
harmful habits (tobacco smokers and tobacco smokers+alcohol drinking habit), CD4 level, use and type of antiretroviral. To minimize the possible errors during the data transfer a standard data model was developed. All information was transferred by two trained researchers.

The diagnosis of OHL was performed based on clinical and histological characteristics.

Univariate analyses were used to identify changes in each variable throughout the study period. For categorical data analysis multiple correspondence analysis were performed to determine the association between qualitative variables. Binary logistic regression was applied to evaluate the HIV patients with OHL and the explanatory variables considered. Then logistic models, namely Pearson, deviation and Hosmer-Lemeshow, were applied and adjusted indicating the significant variables. Then the Cramer $\mathrm{V}$ coefficient was used as correlation ratio. The chi-square test was used to evaluate the impact of antiretroviral therapy (ARVT) on OHL. The results were considered statistically significant at $p<0.05$.

\section{Results}

During the data transfer it was noted that certain records were lacking some information. This fact is reflected in the total number of evaluations in some analysis.

During the period starting from 1989 to 2006, 1600 new HIV-positive patients were admitted and evaluated at the center, OHL was observed in 215 (13.4\%) from those. The first figure (Figure 1) shows the incidence of OHL during the study period, demonstrating a gradual decrease in this lesion expression. It should be noted that 50 new patients were admitted in 2006 and none of them presented with the disease.

Regarding the age of these patients, the vast majority 112 (52.1\%) was in the fourth decade of life. Patients in the third and fifth decades were observed in 63 (29.3\%) and 29 (13.5\%) respectively. In the first, sixth and seventh decades of life only one patient, in each decade, $(0.01 \%)$ was diagnosed with OHL.

Tobacco smoking was the most frequent substance used stated by all the patients studied. Among patients

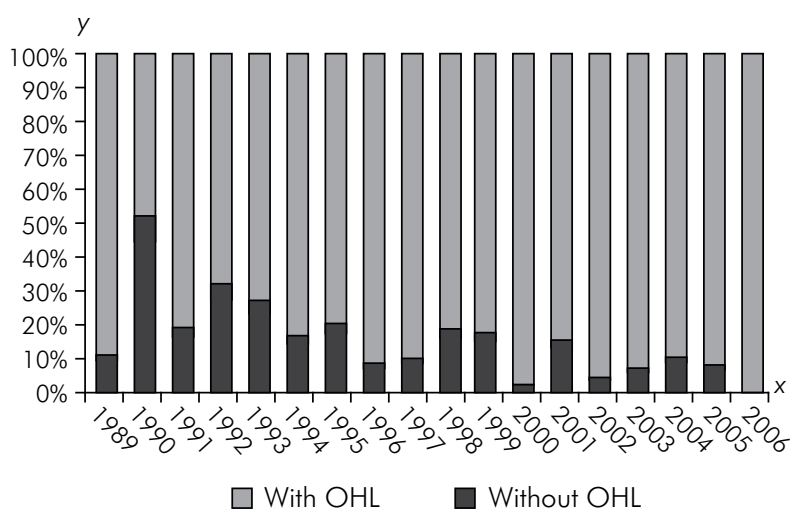

Figure 1. Annual percentage of new and HIV-positive patients who presented hairy leukoplakia in the period 1989. 2006 ( $n=215)$.

with OHL who reported any harmful habits (168); $114(68 \%)$ revealed tobacco and $37(22 \%)$ alcohol use, $10(6 \%)$ smoked marijuana and $5(3 \%)$ and $2(1 \%)$ were addicted to cocaine and crack, respectively.

Among 215 patients with OHL 211 (98.1\%) were conscious of their presumable HIV exposure category. The most frequently reported HIV exposure category was through sexual contact in 168 (79.5\%) of the patients, heterosexual was the most prevalent 85 $(40.5 \%)$ and followed by homosexual contact 70 (33\%) and bisexual 13 (6\%). The injecting drug users (IDUs) were observed in 39 (18\%), those infected through blood transfusion $9(4 \%)$ and children infected vertically 2 (1\%).

Information regarding CD4+ levels at the time of OHL diagnosis was available in 63 (29.3\%) of the patients. OHL occurred mostly in patients with CD4 counts between 200 and 500 cells/ $\mathrm{mm}^{3} 35$ (55.5\%), $16(25.3 \%)$ had counts above 500 cells $/ \mathrm{mm}^{3}$ and 12 (19\%) below 200 cell $/ \mathrm{mm}^{3}$.

Regarding the use of anti-retroviral drugs 110 (51\%) patients were in treatment, while 105 (49\%) did not use any ARVT. Three different classes of antiretroviral drugs were identified among patients in treatment: protease inhibitors (PIs), non-nucleoside reverse transcriptase inhibitors (NNRTIs) and nucleoside reverse transcriptase inhibitors (NRTIs). In general, the treatment regimens used combined 2 different drugs (NRTI+NNRTI or NRTI+PI) and none of the patients using NNRTIs presented PIs in their therapeutic regimen. 
The incidence of OHL between 1989 and 1996 was 16.3\%. In 1996, PIs were introduced systematically in the treatment of HIV patients and reached a peak in 2000. During that period the incidence of OHL showed a reduction reaching $12.6 \%$. A second period of reduction in the incidence of OHL could be identified from 2000 to 2006 with the introduction of NNRTIs, when the incidence of OHL decreased to $7.7 \%$ (Figure 2).

Statistical analysis (chi square) was performed between the two different groups of ARVT (missing data were excluded). Thirteen $(9.9 \%)$ of 131 patients who received NRTI+NNRTI presented OHL compared with 37 (12.2\%) of 304 patients who received NRTI+PI. The patients who received NRTI+PI had a relative risk 0.9 times higher for the disease. Nevertheless, this finding was not statistically significant $(p=0.5)$.

In order to verify the association between the categorical variables during the study period, four sub-periods were established (P1: from 1989 to 1992, P2: from 1993 to 1996, P3: from 1997 to 2000, P4: from 2001 to 2005) and a multiple correspondence analysis was performed.

Figure 3 identifies the patients diagnosed with OHL in P1. This population was characterized by fourth decade males infected by injectable drugs and not under any kind of ARVT or only NRTI.

During P2 and P3 no association was observed. Figure 4 illustrates the P4 interval. In this last sub-period patients with OHL formed a group of men in their fourth decade of life that presented smoking and drinking habits. However, in the same period it is possible to identify a second group composed of women in fourth or fifth decade of life, heterosexual, tobacco smokers and under NRTI+NNRTI therapy.

Specific information with respect to the CD4 cell count was available in 556 charts of 1600 patients. The correlation between the considered variables (ARVT, harmful habits, decade of life, exposure category, CD4 count), according to the V Cramer method, revealed the variable "exposure category" statistically correlated with "decade of life" $(p=0.560)$ and "sex" $(p=0.621)$.

Originally a model was adjusted including the main effect of all variables. The model adjustment resulted in the harmful habits ("tobacco smokers" $(p=0,012)$ and "tobacco smokers+alcohol drinking habit" $(p=0,008))$ and "level of CD4" $(p=0,019)$ as the statistically significant variables. Confidence interval of $95 \%$. Both adjustments presented a descriptive level greater than $5 \%$ for the three tests (Pearson, Deviance and Hosmer-Lemeshow), particularly the final model that achieved a lower value in the chi-square estimation for the three methods, thus indicating a better fit to the data.

From those data analyses it is possible to predict that a patient with a CD4 count less than or equal to $500 \mathrm{cell} / \mathrm{mm}^{3}$ and tobacco smoker has an estimated odds of developing OHL of 2.23 times $(95 \% \mathrm{CI}=1.20-4.17)$. The estimated odds of a patient with CD4 count less than or equal to $500 \mathrm{cell} / \mathrm{mm}^{3}$, smoker and alcohol user of developing OHL is 2.43 (95\% CI $=1.26-4.67]$. And the chance of an individual with CD4 level less than or equal to 500 cells $/ \mathrm{mm}^{3}$ that has no harmful habit presents LP is $2.5(1 / 0$, 4) times the odds of those with CD4 counts greater than 500 cells $/ \mathrm{mm}^{3}$ and has no harmful habit (Table).

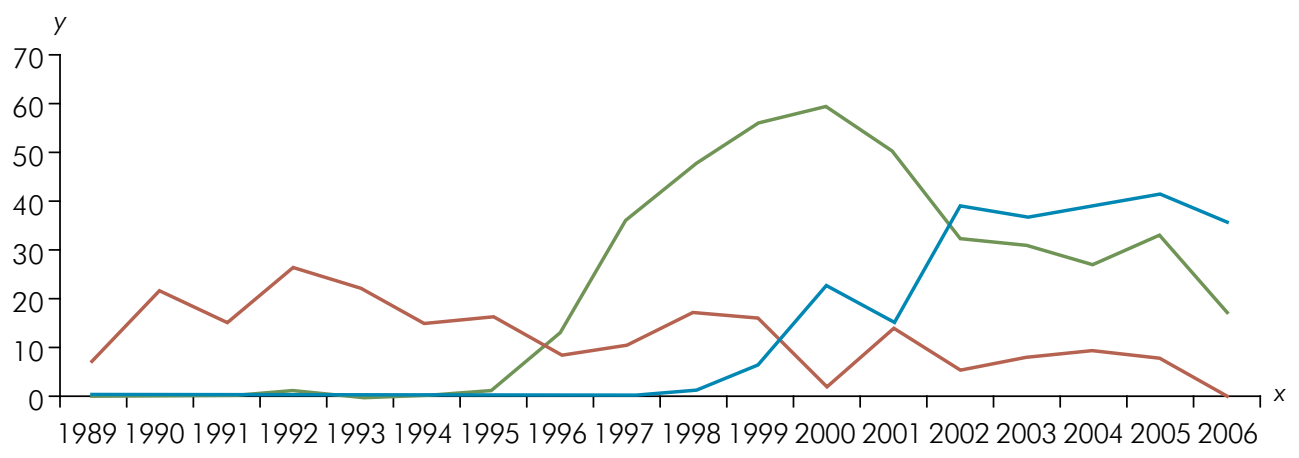

HL

NRTI+NNRTI

$\mathrm{NRTI}+\mathrm{PI}$

Figure 2. Annual incidence of hairy leukoplakia and the type of ARVT used by 1600 HIV-positive patients. 


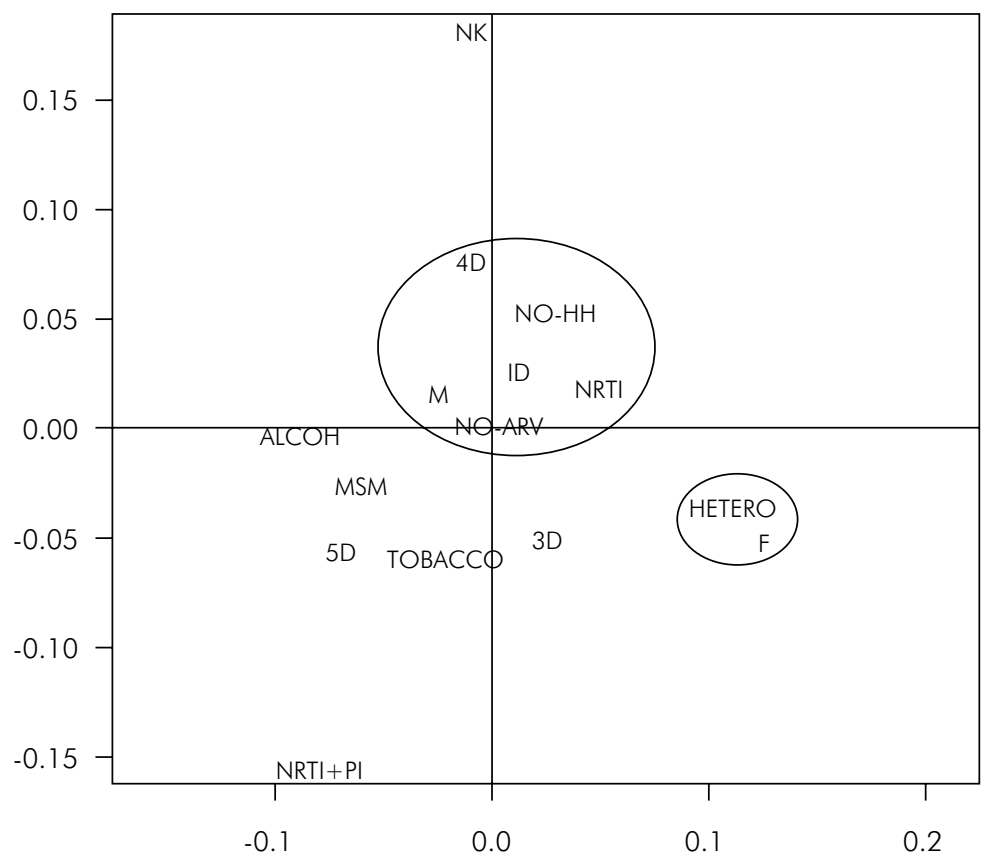

NK: not known; 4D: fourth decade; NO-HH: no harmful habtis; ID: injectable drugs users; M: male; NRTI: nucleoside reverse transcriptase inhibitors; NO-ARV: no antiretroviral; ALCOH: alcohol; MSM: men who have sex with men; HETERO: heterosexual; 5D: fifith decade; TOBACCO: tobacco smokers; 3D: third decade; F: female; NRTI+PI: nucleoside reverse transcriptase inhibitors and protheinase inhibitors.

Figure 3. Correspondence analysis, from 1989 to 1992, lists the variables: age, harmful habits, HIV exposure category, gender and ARV.

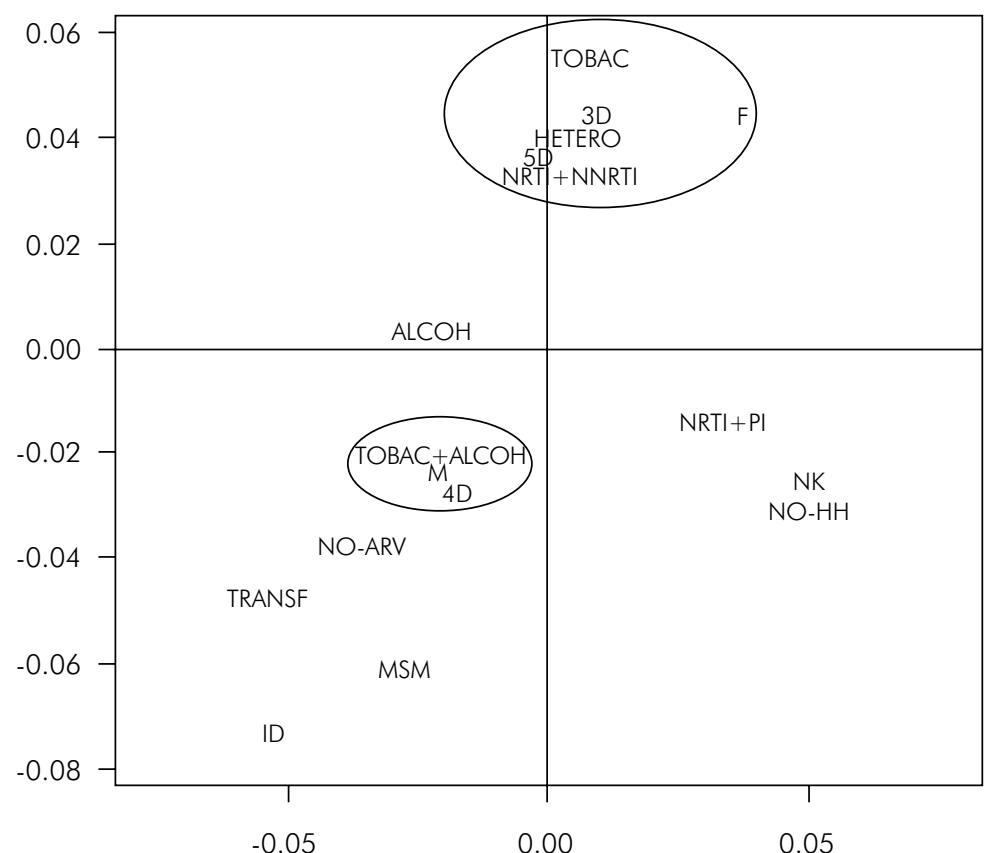

TOBAC: tobacco smokers; 3D: third decade; F: female; HETERO: heterosexual; 5D: fifith decade; NRTI+NNRTI: nucleoside reverse transcriptase inhibitors and non-nucleoside reverse transcriptase inhibitors; ALCOH: alcohol; M: male; 4D: fourth decade; NRTI+PI: nucleoside reverse transcriptase inhibitors and protheinase inhibitors; NK: not known; NO-HH: no harmful habtis; NO-ARV: no antiretroviral; TRANSF: transfusion; MSM: men who have sex with men; ID: injectable drugs users.

Figure 4. Correspondence analysis, from 2001 to 2005, lists the variables: age, harmful habits, HIV exposure category, gender and ARV. 
Table. Estimated odds ratios.

\begin{tabular}{ccccc}
\hline CD4 count & Harmful habits & $\begin{array}{c}\text { Odds } \\
\text { ratio }\end{array}$ & $\begin{array}{c}\text { Limit } \\
\text { inferior }\end{array}$ & $\begin{array}{c}\text { Limit } \\
\text { superior }\end{array}$ \\
\hline \multirow{2}{*}{ CD4 $\leq 500$} & No-hh or alcohol & 1 & 1 & 1 \\
& Tobacco & 2,23 & 1,2 & 4,17 \\
& Tobaco+alcohol & 2,43 & 1,26 & 4,67 \\
CD4 $>400$ & No-hh or alcohol & 0,4 & 0,18 & 0,86 \\
& Tobaco & 0,88 & 0,87 & 6,7 \\
& Tobaco+alcohol & 0,96 & 0,94 & 7,23 \\
\hline
\end{tabular}

No-hh: no harmful habits.

\section{Discussion}

There are numerous studies about the prevalence of oral manifestations associated with HIV-infection and there is a general consensus regarding the decrease in these oral lesions with the introduction of HAART. However, the oral cavity is still considered as a common site for the occurrence of opportunistic lesions in HIV-infected patients on ARVT. In addition, it has been suggested that the presence of oral lesions might be not only predictors of disease progression but also a clinical predictor of HAART failure or non-adherence ${ }^{12,16}$.

One of the limitations of this study is related to the study design. In cross-sectional analytical epidemiological studies is not known whether exposure proceeds or is a consequence of the disease/condition related to health.

The diagnosis of OHL is still a controversial issue, specially the necessity of the association between clinical aspects, histopathological/cytopathological features and EBV molecular detection. The gold-standard to stablish the final diagnosis of OHL is the detection of EBV in epithelial cells by in situ hybridization ${ }^{8,9}$. Some authors have used PCR to EBV detection, but is not a good tool to stablish the epithelial infection because of the saliva contamination in biopsy or cytological samples ${ }^{8,9}$. In known HIV-seropositive patients the OHL diagnosis can be stablished by an experienced clinician based on clinical and histopathological features ${ }^{3}$.

The demographic characteristics reported in the literature suggest a higher prevalence of OHL among HIV positive males, particularly homosexuals ${ }^{1,18,19,20,21}$. Our study likewise showed the male gender as the most affected, though the incidence of OHL among women had increased in the latest years. Contrary to some studies the most prevalent exposure risk category in patients with OHL was the heterosexual. Also the age group of patients with OHL suffered a variation over the years. The fourth decade of life was the most prevalent however we noticed that this age group has decreased as a consequence of the increase patients in the fifth decade of life. Although related data concerning longitudinal studies are sparing this differences may be related to the HIV epidemic progression.

The HIV epidemic profile in Brazil has been experiencing dramatics changes in the past decade. Markedly the increased amount of infected women, the increment in age of the HIV-positive population, and the crescent group exposed through heterosexual contact $^{17}$. These facts can contribute for the changes in epidemiological profile of patients with OHL in the present study.

Statistically elevated risk of OHL in smokers compared with nonsmokers has been noted in this study and is in agreement with previously published data ${ }^{15,20,22,23}$. Cigarette smoking has already been established as a strong risk factor for oral cancer. Even though there is no evidence of OHL being a premalignant lesion, it is possible that oral mucosa become more susceptible to infection by EBV, influenced by cigarettes chemical compounds ${ }^{23}$.

Regarding laboratorial parameters, several studies demonstrated the relation between the presence of OHL and a lower CD4+ account ${ }^{15,18,24}$ but no association was found between HIV viral load and opportunistic infections ${ }^{25}$. However, some authors believe that, despite this correlation, OHL can occur regardless of the CD4 level ${ }^{20,22}$. In the present study the highest incidence of OHL was among patients with CD4 counts range from 200 to 500 cells $/ \mathrm{mm}^{3}$ (55.5\%).

During the study period, a reduction of OHL cases (Figure 1) was observed suggesting an improvement in the immune status of HIV patients over the years. This enhancement can be credited to the systematic use of ARVT experienced since 1996, period when the government began the free distribution of ARVs to all patients in need of medication ${ }^{26}$. Regarding the use of ARVs, we were also able to verify the efficacy 
of different classes of drugs in the prevention of OHL. The largest number of patients with OHL was those who did not use any medication or not combined different classes of drugs in their regimen. In our study, the correlation between a lower incidence of OHL in patients taking NNRTI could be observed, but statistical tests were not favorable to this association, patients under NNRTI therapy showed an increase of OHL in the last decade (Figure 4). This apparent contradictory outcome may be explained by the adaptations of ARV recommended therapy implemented by Brazilian Ministry of Health. According to the 1997 recommendation the therapeutic regimen must include 2 NRTI and in some cases a triple regimen might be used with the addition of one $\mathrm{P}^{27}$, this therapy was applied until 1999 when based on recent advances in the area a new strategy was recommended replacing the triple therapy with PI by the triple with NNRTI ${ }^{28}$ thus consequently increasing the number of patients taking NNRTI.

There is some controversy in the literature regarding the effectiveness of ARVT in reducing the incidence of OHL. Although some studies demonstrate the effectiveness of ARV drugs ${ }^{16,29}$, especially PIs ${ }^{14,16}$, others did not observe this association ${ }^{15,19,24}$.

This issue can and should be studied further in future, since recent regimens containing NNRTI or

\section{References}

1. Ranganathan K, Umadevi M, Saraswathi TR, Kumarasamy N, Solomon S, Johnson N. Oral lesions and conditions associated with human immunodeficiency virus infection in 1000 South Indian patients. Ann Acad Med Singapore. 2004;33(4 Suppl):37-42.

2. Coogan MM, Greenspan J, Challacombe SJ. Oral lesions in infection with human immunodeficiency virus. Bull World Health Organ. 2005;83(9):700-6. doi:10.1590/S0042-96862005000900016

3. Pindborg JJ, Williams DM. [An update of the classification and of the diagnostic criteria of oral lesions in HIV infection. Minerva Stomatol. 1993;42(5):223-7.

4. Lourenço AG, Motta AC, Figueiredo LT, Machado AA, Komesu MC. Oral lesions associated with HIV infection before and during the antiretroviral therapy era in Ribeirão Preto, Brazil. J Oral Sci. 2011;53(3):379-85. doi:10.2334/josnusd.53.379
NNRTI + PI, have been associated with a greater virologic suppression thereby promoting immunologic reconstitution comparing to regimens containing only $\mathrm{PIs}^{30}$. On the other hand, there is a great number of different antiretroviral drugs classes such as Integrase Inhibitors, Fusion/Entry Inhibitors and Multidrug Combination Products that has been introduced in the HIV management and can also have an effect on prevalence of oral manifestations.

\section{Conclusion}

The study allowed us to conclude that $\mathrm{OHL}$ is a clinical manifestation of the immunodeficiency induced by HIV infection and is related to the patient CD4 count, use of ARVT and tobacco use is also more prevalent in men with heterosexual behavior in the fourth decade of life.

These characteristics were recognized in absolute values, but when verifying the behavior over the years we noticed that the incidence of OHL is decreasing and its epidemiological characteristics changing. The frequency of this lesion has increased in female patients in the fifth decade of life.

The decrease in incidence of OHL appears to be linked to immune restoration induced by ARVT and the changes in Brazilian HIV epidemic seems to play an important role in the demographic characteristics of OHL.

5. Greenspan D, Greenspan JS. Significance of oral hairy leukoplakia. Oral Surg Oral Med Oral Pathol. 1992;73(2):151-4. doi:10.1016/0030-4220(92)90187-U

6. Piperi E, Omlie J, Koutlas IG, Pambuccian S. Oral hairy leukoplakia in HIV-negative patients: report of 10 cases. Int J Surg Pathol. 2010;18(3):177-83. doi:10.1177/1066896908327865

7. Resnick L, Herbst JS, Raab-Traub N. Oral hairy leukoplakia. J Am Acad Dermatol. 1990;22(6 Pt 2):1278-82. doi:10.1016/0190-9622(90)70174-G

8. Braz-Silva PH, de Rezende NP, Ortega KL, de Macedo Santos RT, Magalhães MH. Detection of the Epstein-Barr virus (EBV) by in situ hybridization as definitive diagnosis of hairy leukoplakia. Head Neck Pathol. 2008;2(1):19-24. doi:10.1007/s12105-007-0039-9

9. Braz-Silva PH, Santos RT, Schussel JL, Gallottini M. Oral hairy leukoplakia diagnosis by Epstein-Barr virus in situ hybridization in liquid-based cytology. Cytopathology. 2014;25(1):21-6. doi:10.1111/cyt.12053 
10. 1993 revised classification system for HIV infection and expanded surveillance case definition for AIDS among adolescents and adults. MMWR Recomm Rep. 1992;41(RR-17):1-19.

11. Ramírez-Amador V, Anaya-Saavedra G, Calva JJ, Clemades-Pérez-de-Corcho T, López-Martínez

C, González-Ramírez I et al. HIV-related oral lesions, demographic factors, clinical staging and anti-retroviral use. Arch Med Res. 2006;37(5):646-54. doi:10.1016/j.arcmed.2006.01.002

12. Miziara ID, Weber R. Oral candidosis and oral hairy leukoplakia as predictors of HAART failure in Brazilian HIV-infected patients. Oral Dis. 2006;12(4):402-7. doi:10.1111/j.1601-0825.2005.01214.x

13. Baccaglini L, Atkinson JC, Patton LL, Glick M, Ficarra G, Peterson DE. Management of oral lesions in HIV-positive patients. Oral Surg Oral Med Oral Pathol Oral Radiol Endod. 2007;103 Suppl:S50 e1-23. doi:10.1016/j.tripleo.2006.11.002

14. Patton LL, McKaig R, Strauss R, Rogers D, Eron JJ, Jr. Changing prevalence of oral manifestations of human immuno-deficiency virus in the era of protease inhibitor therapy. Oral Surg Oral Med Oral Pathol Oral Radiol Endod. 2000;89(3):299-304. doi:10.1016/S1079-2104(00)70092-8

15. Chattopadhyay A, Caplan DJ, Slade GD, Shugars DC, Tien HC, Patton LL. Incidence of oral candidiasis and oral hairy leukoplakia in HIV-infected adults in North Carolina. Oral Surg Oral Med Oral Pathol Oral Radiol Endod. 2005;99(1):39-47. doi:10.1016/j.tripleo.2004.06.081

16. Ortega KL, Vale DA, Magalhães MH. Impact of PI and NNRTI HAART-based therapy on oral lesions of Brazilian HIV-infected patients. J Oral Pathol Med. 2009;38(6):489-94. doi:10.1111/j.1600-0714.2009.00783.x

17. Joint United Nations Programme on HIV/AIDS - UNAIDS. AIDS epidemic update. Geneva: Unaids; 2006. http://data. unaids.org/pub/EpiReport/2006/2006_EpiUpdate_en.pdf

18. Chattopadhyay A, Caplan DJ, Slade GD, Shugars DC, Tien HC, Patton LL. Risk indicators for oral candidiasis and oral hairy leukoplakia in HIV-infected adults. Community Dent Oral Epidemiol. 2005;33(1):35-44. doi:10.1111/j.1600-0528.2004.00194.x

19. Kerdpon D, Pongsiriwet S, Pangsomboon K, Iamaroon A, Kampoo K, Sretrirutchai S, et al. Oral manifestations of HIV infection in relation to clinical and CD4 immunological status in northern and southern Thai patients. Oral Dis. 2004;10(3):138-44. doi:10.1046/j.1601-0825.2003.00990.x
20. Patton LL, McKaig RG, Strauss RP, Eron JJ, Jr. Oral manifestations of HIV in a southeast USA population. Oral Dis. 1998;4(3):164-9. doi:10.1111/j.1601-0825.1998.tb00274.x

21. Dongo M, Gonçalves LS, Ferreira SM, Noce CW, Dias EP, Silva Junior A. Gender differences in oral manifestations among HIV-infected Brazilian adults. Int Dent J. 2013;63(4):189-95. doi:10.1111/idj.12029

22. Sroussi HY, Villines D, Epstein J, Alves MC, Alves ME. Oral lesions in HIV-positive dental patients: one more argument for tobacco smoking cessation. Oral Dis. 2007;13(3):324-8. doi:10.1111/j.1601-0825.2006.01289.x

23. Shiboski CH, Neuhaus JM, Greenspan D, Greenspan JS. Effect of receptive oral sex and smoking on the incidence of hairy leukoplakia in HIV-positive gay men. J Acquir Immune Defic Syndr. 1999;21(3):236-42. doi:10.1097/00126334-199907010-00009

24. Greenspan D, Gange SJ, Phelan JA, Navazesh M, Alves $\mathrm{ME}$, MacPhail LA, et al. Incidence of oral lesions in HIV-1-infected women: reduction with HAART. J Dent Res. 2004;83(2):145-50. doi:10.1177/154405910408300212

25. Sempa JB, Dushoff J, Daniels MJ, Castelnuovo B, Kiragga AN, Nieuwoudt $\mathrm{M}$ et al. Reevaluating cumulative HIV-1 viral load as a prognostic predictor: predicting opportunistic infection incidence and mortality in a Ugandan Cohort. Am J Epidemiol. 2016;184(1):67-77. doi:10.1093/aje/kwv303

26. Galvao J. Access to antiretroviral drugs in Brazil. Lancet. 2002;360(9348):1862-5. doi:10.1016/S0140-6736(02)11775-2

27. Ministério da Saúde (BR). SdVeS, Departamento de DST, AIDS e Hepatites Virais. Recomendações para terapia antirretroviral em adultos e adolescentes infectados pelo HIV: 1997. Brasília, DF: Ministério da Saúde;1997.

28. Ministério da Saúde (BR). Departamento de DST, AIDS e Hepatites Virais. Recomendações para terapia anti-retroviral em adultos e adolescentes infectados pelo HIV: 1999. Brasília, DF: Ministério da Saúde;1999.

29. Moura MD, Grossmann SM, Fonseca LM, Senna MI, Mesquita RA. Risk factors for oral hairy leukoplakia in HIV-infected adults of Brazil. J Oral Pathol Med. 2006;35(6):321-6. doi:10.1111/j.1600-0714.2006.00428.x

30. Bartlett JA, Fath MJ, Demasi R, Hermes A, Quinn J, Mondou E et al. An updated systematic overview of triple combination therapy in antiretroviral-naive HIV-infected adults. AIDS. 2006;20(16):2051-64. doi:10.1097/01.aids.0000247578.08449.ff 